\title{
An implementation of web based discrete event simulation using Core Manufacturing Simulation Data (CMSD): A case study
}

\author{
Web based discrete event simulation
}

\author{
Gopal Kumar, Xiao Liu, Paul Young, John Geraghty, Ganesh Pardeshi
}

\begin{abstract}
The cost of developing, implementing, using simulation technology, and integrating them is very high. There are efforts to promote interoperability and facilitate data exchange among various business process systems conveniently and efficiently. The international Simulation Interoperability Standards Organization (SISO) developed and standardized the Core Manufacturing Simulation Data (CMSD) specification to foster the more widespread use of manufacturing technology through the reduction of data interoperability issues. The CMSD provides information model describing characteristics of and relationships between the core manufacturing entities which in turn can be used for simulating manufacturing systems. The paper through a case demonstrates an example using eVSM and CMSD document to integrate real world manufacturing based application. The paper concludes by highlighting the fact that CMSD document coupled with a standalone DES application has great potential to reduce cost, time, and expertise required to carry DES which will be immensely beneficial for small and medium enterprises (SMEs).
\end{abstract}

Keywords - CMSD, simulation, modelling, interoperability, eVSM

\section{Introduction}

This Discrete event simulation (DES) is one of the most commonly used methods to analysing and understanding the complex and dynamic manufacturing systems (Tako and Robinson, 2010). It is a highly effective tool which enables us to evaluate operating strategies and various alternative configurations to support decision making. The DES and its benefits in key performance metrics are acknowledged widely (Jahangirian et al., 2010). Commercial off-the-self DES software traditionally contributed immensely to the diffusion of DES. However, such software comes with multidimensional cost which deters companies, especially SMEs, from adapting DES based solution in their decision making approach (McLean and Leong, 2001). Even after the advancement of information technology, the present market acceptance of DES among small and medium enterprises (SMEs) is relatively low. The reasons behind the low acceptance of DES include lack of awareness in relation to the potential of DES, supply problem (shortage of delivering DES solutions) and the need of understanding DES in line with the modern manufacturing theory.

For SMEs, who buy annual licences for simulation software, cost involved in the construction of suitable model using data defining current state of affairs and the time required to undertake the multiplicity of simulation runs and subsequent analysis of the large volume of data are factors which significantly limit the use of DES by SMEs. As interoperability is a major issue for communicating different business applications (Bloomfield et al., 2012), the Core Manufacturing Simulation Data (CMSD) standard chartered by the Simulation Interoperability Standards Organization (SISO) to facilitate simulation process and transferring relevant data conveniently. Since then, researchers and practitioners are attempting to use CMSD based data structure to facilitate DES based decision making and for other applications (Barlas et al., 2014; Bloomfield et al., 2012; Fournier, 2011; Johansson et al., 2007). This experiment aims to provide a viable solution to address some of the limitations of SMEs. The Dublin City University (DCU) in partnership with the University of Edinburgh and the Irish Centre for Manufacturing Research (ICMR) has been developing an open source approach to simulation modelling using data driven model structure. The aim is to provide the access to the web-based data driven DES modelling tool which will significantly help SMEs in terms of expertise, cost, and time. Section II presents a test case, and Section III concludes the paper.

\section{A test case}

A simple production flow is created using electronic value stream mapping (eVSM) plugin for Visio which is part of the Microsoft office. The eVSM is a tool for drawing value stream mapping (VSM) electronically. The eVSM website says it is the easiest way to capture, share, analyse, and improve value stream. The VSM can be proven very useful for simulation as it intends to capture and visualise entire production process, representing both information and material flow. The VSM captures information at individual stations about its cycle time, utilization of resources, set-up time, work-in-process inventory, man power requirement, and the information flow (Lian and Landeghem, 2007; Singh et al., 2011).

Production flow was drawn for single part. The production flow contained six processes in series with processing time associated with each of the processes. Each step had pre-processing steps defined. Through this VSM diagram drawn using eVSM, a datasheet can be saved in excel sheet. The datasheet presents production flow in row which includes all process steps and its intermediate buffer, and for each process and buffer its relevant parameters such as cycle time, inventory, etc., are presented in column. This datasheet is processed to generate CMSD compliant XML and then it is used to find out warm-up time and perform simulation run. Identification of warm-up time helps running simulation for a length which can produce satisfactory or reliable results. Finally, actual experiment is run for appropriate simulation length and number of replications which produces simulation results. The results are presented 
Proc. of the Fourth Intl. Conf. Advances in Computing, Communication and Information Technology- CCIT 2016

Copyright (C) Institute of Research Engineers and Doctors, USA .All rights reserved.

ISBN: 978-1-63248-092-7 doi: 10.15224/ 978-1-63248-092-7-47

in a way which can easily be comprehended. The result is presented in Fig. 1.

Results in Fig. 1 are organised into three parts. First, in pictorial form it describes how much each process station is operating, under-repair, and blocked. For example, in Figure 1, RC A0300 MachineC 3805476 is operating $74 \%$ of the time and then it is blocked. Second, it presents part statuscycle time, throughput, and scrap. Mean, standard deviation, and mean with $95 \%$ confidence interval are computed for cycle time, throughput, and scrap. Third, machine status for each replication is presented. For each machine, its utilization, blockage, downtime, and idle time are described.

Figure 1 - Analysis results
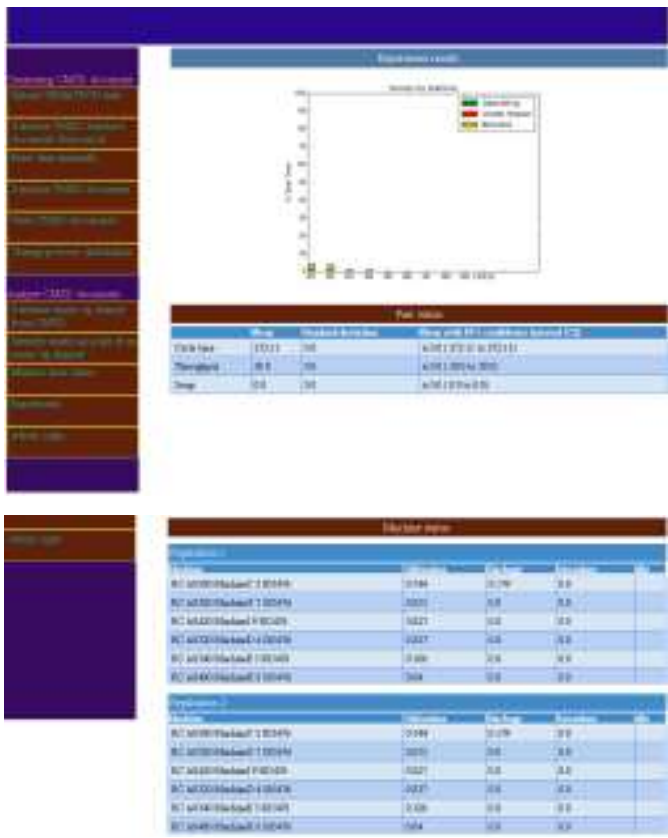

\section{Conclusions}

The aim of this paper is to propose and demonstrate a data driven, web-based, DES systems in order to streamline manufacturing activities, data organisations, simulation process, and interpretation of simulation results. A case demonstrating the use of the system and final results are discussed and presented. The case discussed using eVSM and CMSD document to integrate real world manufacturing systems.

As the systems streamlines data processing, model building, and data communication (CMSD based document provides interoperability for different applications), it provides great opportunities, especially for SMEs, to reduce traditional cost of simulating manufacturing systems and for non-expert to carry and understand analysis.

\section{Acknowledgment}

We are thankful to the Enterprise Ireland who funded this project under the contract CC20101003A. We are also thankful to other organisations and individuals who supported us in various ways in completing the project.

\section{References}

[1] A. A. Tako and S. Robinson, "Model development in discrete-event simulation and system dynamics: An empirical study of expert modellers," Eur. J. Oper. Res., vol. 207, no. 2, pp. 784-794, Dec. 2010 .

[2] M. Jahangirian, T. Eldabi, A. Naseer, L. K. Stergioulas, and T. Young, "Simulation in manufacturing and business: A review," Eur. J. Oper. Res., vol. 203, no. 1, pp. 1-13, May 2010.

[3] C. McLean and S. Leong, "The expanding role of simulation in future manufacturing," in Proceedings of the 33nd conference on Winter simulation, 2001, pp. 1478-1486.

[4] R. Bloomfield, E. Mazhari, J. Hawkins, and Y.-J. Son, "Interoperability of manufacturing applications using the Core Manufacturing Simulation Data (CMSD) standard information model," Comput. Ind. Eng., vol. 62, no. 4, pp. 1065-1079, May 2012.

[5] P. Barlas, G. Dagkakis, C. Heavey, B. Gaffney, P. Young, and J. Geraghty, "Test Implementation and Initialisation of a Simulation Model Using CMSD," Procedia CIRP, vol. 25, pp. 276-282, 2014.

[6] J. Fournier, "Model building with Core Manufacturing Simulation Data," in Simulation Conference (WSC), Proceedings of the 2011 Winter, 2011, pp. 2214-2222.

[7] M. Johansson, B. Johansson, A. Skoogh, S. Leong, F. Riddick, Y. T. Lee, G. Shao, and P. Klingstam, "A test implementation of the core manufacturing simulation data specification," in Proceedings of the 39th conference on Winter simulation: 40 years! The best is yet to come, 2007, pp. 1673-1681.

[8] Y.-H. Lian and H. V. Landeghem, "Analysing the effects of Lean manufacturing using a value stream mapping-based simulation generator," Int. J. Prod. Res., vol. 45, no. 13, pp. 3037-3058, Jul. 2007.

[9] B. Singh, S. K. Garg, and S. K. Sharma, "Value stream mapping: literature review and implications for Indian industry," Int. J. Adv. Manuf. Technol., vol. 53, no. 5-8, pp. 799-809, Mar. 2011.

About Author (s):

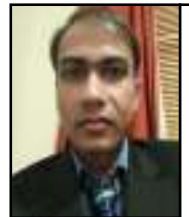

Gopal Kumar received his $\mathrm{PhD}$ in supply chain management and he has been doing research under postdoctoral fellowship at the Dublin City University. He is working on facilitating easy access of advanced methods for improving productivity of SMEs. His research articles have been accepted for publication in Journal of Business and Industrial Marketing, IIMB Management Review, International Journal of Productivity and Performance Management, Benchmarking: an International Journal, etc. His primary research interests include supply chain management, sustainable and green supply chain collaboration, operations management, game theory application in business and management, business process automation and information technology application. Gopal Kumar is the corresponding author and can be contacted at: 